\title{
E-Health System for Medical Telesurveillance of Chronic Patients
}

\author{
C. Rotariu, H. Costin, I. Alexa, G. Andruseac, V. Manta, B. Mustata
}

Cristian Rotariu, Hariton Costin

1. "Gr. T. Popa" Univ. of Medicine and Pharmacy

Kogalniceanu No. 9-13, Iasi, Romania and

2. Institute for Computer Science, Romanian Academy

Carol I No. 11, Iasi, Romania

E-mail: crotariu74@yahoo.com, hcostin74@yahoo.com

\section{Ioana Alexa, Gladiola Andruseac}

"Gr. T. Popa" Univ. of Medicine and Pharmacy,

Kogalniceanu No. 9-13, Iasi, Romania E-mail: agladi@yahoo.com

\section{Vasile Manta}

"Gh. Asachi" Technical University,

D. Mangeron No. 27, Iasi, Romania

E-mail:vmanta@cs.tuiasi.ro

\section{Bogdan Mustata}

ROMSOFT Ltd.

Sulfinei No. 18, Iasi, Romania

E-mail: mbo@rms.ro

\begin{abstract}
The current common goal in medical information technology today is the design and implementation of telemedicine solutions, which provide to patients services that enhance their quality of life. Advances in wireless sensor network technology, the overall miniaturization of their associated hardware low-power integrated circuits and wireless communications have enabled the design of low-cost, miniature, and intelligent physiological sensor modules with applications in the medical industry. These modules are capable of measuring, processing, communicating one or more physiological parameters, and can be integrated into a wireless personal area network. This paper is dedicated to the most complex Romanian telemedical pilot project, TELEMON, which has as goals design and implementation of an electronic-informaticstelecommunications system, that allows the automatic and complex telemonitoring, everywhere and every time, in (almost) real time, of the vital signs of persons with chronic illnesses, of elderly people, of those having high medical risk and of those living in isolated regions. The final objective of this pilot project is to enable personalized medical teleservices delivery, and to act as a basis for a public service for telemedical procedures in Romania and abroad.

Keywords: telemedicine, telemonitoring, biomedical devices, wireless personal area network.
\end{abstract}

\section{Introduction}

Telemedicine is part of the expanding use of communications technology in health care and is used in prevention, disease management, home health care, long-term care, emergency medicine, and other applications.

The proposed system, called TELEMON, enables to design a secure digital transmission (medical records, digital images, video, and text) and a secure medical records acquisition system in order to enhance the telemedical consultancy services. The main objective of this project 
is to enable personalized teleservices delivery and patient safety enhancement based on an earlier diagnosis with medical telemetry using biosignals, images [1], text transmissions, and also applying the suitable treatment according to the remote medical experts' recommendations [2].

Our project allows persons with different (chronic) diseases and to elderly/lonely people to be monitored from medical and safety points of view. In this way the medical risks and accidents will be diminished. The TELEMON system will act as a pilot project destined to the implementation of a public e-health service, "everywhere and every time", in real time, for people being in different hospitals, at home, at work, during the holidays, on the street, etc.

\section{Materials and Methods}

The main objective of this project is the achievement of an integrated system, mainly composed by the following components in a certain area: a personal network of wireless transducers (PNWT) on the ill person (Figure 1), a data multiplexing block and a personal server (PS) in form of a Personal Digital Assistant (PDA). After local signal processing, according to the specific monitored feature, the salient data are transmitted via one of internet or GSM/GPRS to the database server of the Regional Telemonitoring Centre. The PNWT includes medical devices for vital signs (ECG, heart rate, arterial pressure, oxygen saturation, body temperature), a fall detection module, a respiration one, all these components having radio micro-transmitters, which allows an autonomic movement of the subject. The data processing will be performed by the PDA.

The results of data processing are in principal and if necessary different locally generated alarms, transmitted to the central server. Other results on server data processing will be different medical statistics, necessary for the evaluation of health status of the subject, for the therapeutic plan and for the healthcare entities.

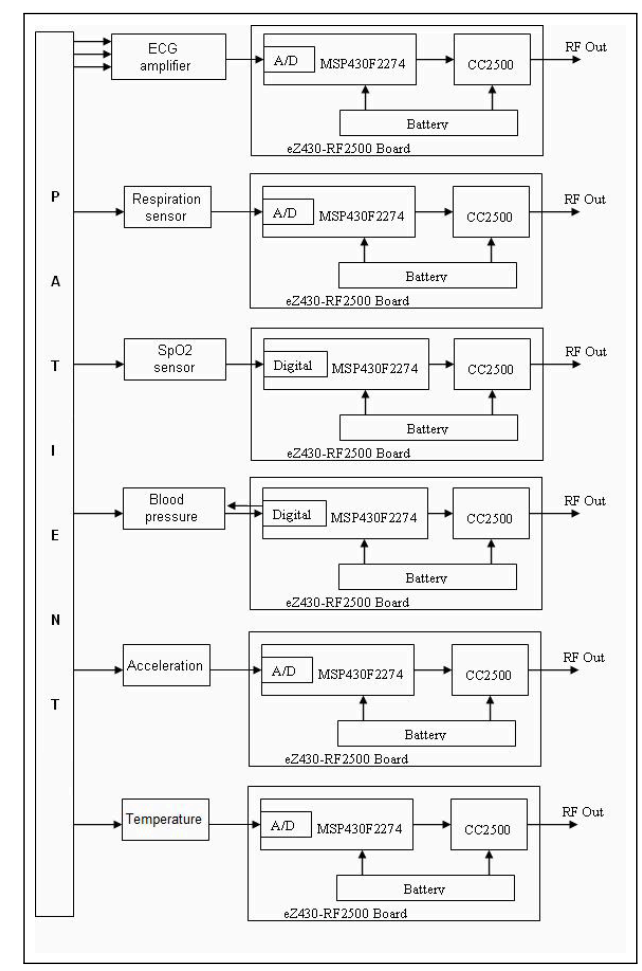

Figure 1: The local subsystem for home monitoring of the patient 
a) a 3-leads ECG module records and transmits data through a radio transceiver interface;

b) the oxygen saturation module ( $\mathrm{SpO} 2)$, that also computes the cardiac rhythm;

c) the arterial pressure module, with serial interface;

d) the body temperature module;

e) the respiration module;

f) the fall detection module.

The modules (a), (d), (e) were made by our research team, while module (b), (c), and (f) were chosen from the market and were integrated in TELEMON system.

These modules transmit data to a PDA through radio transceivers, operate in the $2.4 \mathrm{GHz}$ band, and have $5 \mathrm{~m} / 10 \mathrm{~m}$ range indoors/outdoors.

Our wireless personal area network is realized by using a custom developed sensors modules for physiologic parameters measurement and a low power microcontroller board (eZ430-RF2500 Board from Texas Instruments). The network is wirelessly connected to a personal server that receives the information from sensors.

The eZ430-RF2500 is a complete MSP430 [17] wireless development tool providing all the hardware and software for the MSP430F2274 microcontroller and CC2500 $2.4 \mathrm{GHz}$ wireless transceiver [18]. Operating on the $2.4 \mathrm{GHz}$ unlicensed industrial, scientific and medical (ISM) bands, the CC2500 provides extensive hardware support for packet handling, data buffering, burst transmissions, authentication, clear channel assessment and link quality. The radio transceiver is also interfaced to the MSP430 microcontroller using the serial peripheral interface.

The 3-lead ECG amplifier (Figure 2) is a custom-made device. It has for each channel a gain of 500, is DC coupled and has a cut-off frequency around $35 \mathrm{~Hz}$. The high common mode rejection $(>90 \mathrm{~dB})$, high input impedance $(>10 \mathrm{M} \Omega)$, the fully floating patient inputs are other features of the ECG amplifier.

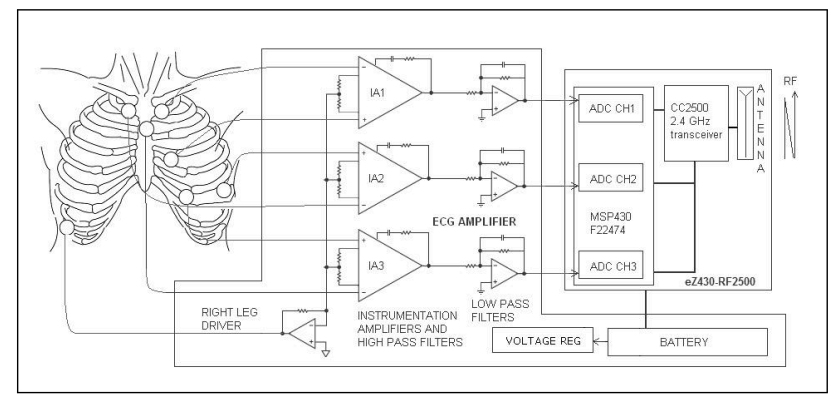

Figure 2: The ECG amplifier (block diagram)

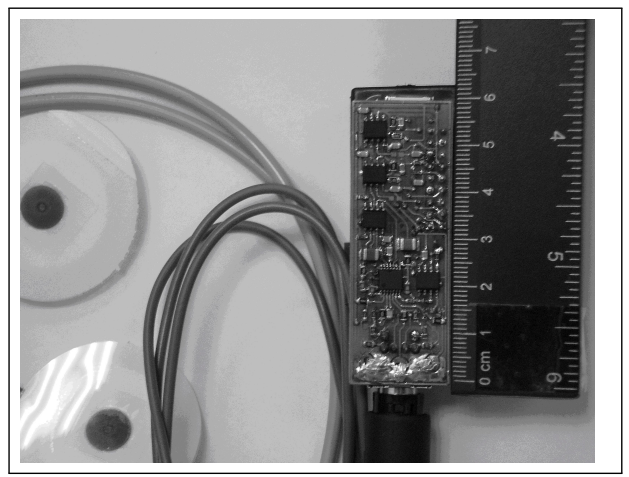

Figure 3: The 3-leads ECG module 
Two AAA 1.2V rechargeable batteries power the ECG amplifier through a voltage regulator. The regulator is built around a capacitive DC/DC step-up converter.

The process of recognition of the ECG waves (Figure 4) constitutes a significant part of the most ECG analysis systems. In applications were rhythm detection is performed, only the location of the $\mathrm{R}$ wave is required. In other applications it is necessary to find and recognize the features of the ECG signal, such as the P and T waves, or the ST segment, for the automated classification and diagnosis. Many algorithms for the extraction of the ECG features based on the digital filters have been reported in the literature [13], [14] and [15] especially algorithms for the QRS complex recognition. The main effort in the ECG features extraction is for finding the exact location of the waves. After that, the determination of the wave's amplitudes and shapes is much simpler. The strategy for finding the exact location of the waves is to first filter the ECG signal and then recognize the QRS complexes. The baseline and the ST segment features are also computed.

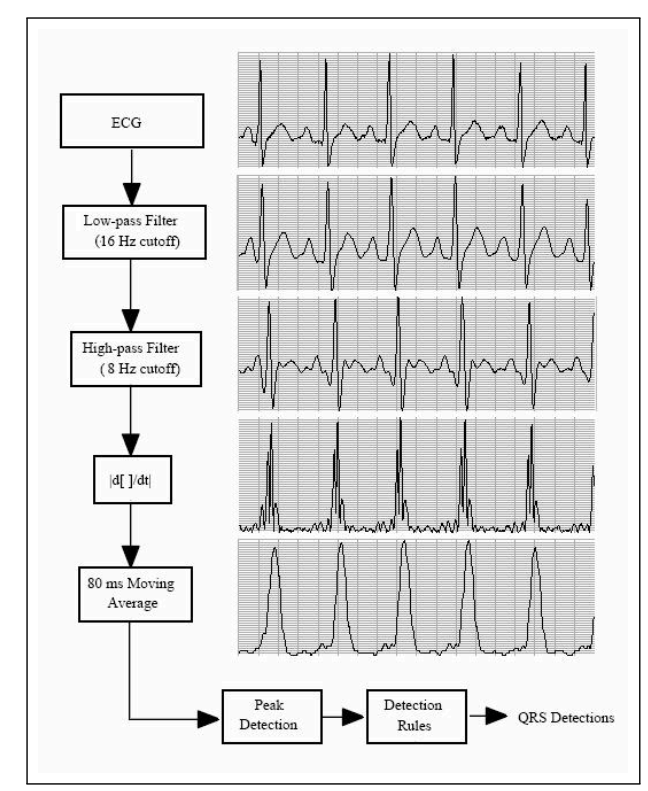

Figure 4: The ECG processing flowchart

The ECG preprocessing stage uses the raw signal to generate a windowed estimate of the energy in the QRS frequency band by using the following filters:

- Low pass filter;

- High pass filter;

- Taking the absolute value of the derivative;

- Averaging the absolute value over an $80 \mathrm{~ms}$ window.

The combined highpass, lowpass and derivative filters produces a bandpass filter with the bandwidth that contains most of the energy in the QRS complex. The theory and implementation of these filters are detailed in [15]. The averaging window was chosen to be the width of a typical QRS complex (80ms).

After the signal ECG filtering, the algorithm detects peaks in the signal. Each time a peak is detected it is classified as either a QRS complex or noise, or it is saved for later classification. The algorithm uses the peak height, peak location (relative to the last QRS peak), and maximum derivative to classify peaks.

The classification algorithm [16] uses the following rules:

- all peaks that precede or follow larger peaks by less than $200 \mathrm{~ms}$ are ignored; 
- if a peak occurs, check to see whether the raw signal contained both positive and negative slopes. If not, the peak represents a baseline shift;

- if the peak occurred within $360 \mathrm{~ms}$ of a previous detection check to see if the maximum derivative in the raw signal was at least half the maximum derivative of the previous detection. If not, the peak is assumed to be a T-wave;

- if the peak is larger than the detection threshold call it a QRS complex, otherwise call it noise.

- if no QRS has been detected within 1.5 R-to-R intervals, there was a peak that was larger than half the detection threshold, and the peak followed the preceding detection by at least 360 ms, classify that peak as a QRS complex.

The detection threshold used in the last two rules is calculated using estimates of the QRS peak and noise peak heights. Every time a peak is classified as a QRS complex, it is added to a buffer containing the eight most recent QRS peaks. Every time a peak occurs that is not classified as a QRS complex, it is added to a buffer containing the eight most recent non-QRS peaks (noise peaks). The detection threshold is set between the average of the noise peak and QRS peak buffers according to the formula:

Det_Th $=$ Avg_Noise_Peak $+\mathrm{TH}^{*}($ Avg_QRS_Peak - Avg_Noise_Peak $)$,

where TH is the threshold coefficient. Similarly, the R-to-R interval estimate used in last rule is computed as the average of the last eight $\mathrm{R}$-to- $\mathrm{R}$ intervals.

The Personal server receives the signal from the ECG module at $200 \mathrm{~Hz}$ and computes the status of the patient for the following ECG parameters:

- Tachycardia if HR > 140bpm;

- Bradycardia if HR $<45 \mathrm{bpm}$;

- Asistola if HR = 0 bpm for at least 3 sec.;

- ST segment elevation if ST > 200l'V;

- ST segment depression if ST $<-150 \mathrm{l} V$;

- Wider QRS if QRS duration >0,12 sec.

For the body temperature measurement we use the TMP275 temperature sensor (Texas Instruments). The TMP275 is a $0.5^{\circ} \mathrm{C}$ accurate, two-wire, serial output temperature sensor available in an SO8 package. The TMP275 is capable of reading temperatures with a resolution of $0.0625^{\circ} \mathrm{C}$. The TMP275 is directly connected to the ez430-RF2500 using the I2C bus and requires no external components for operation except for pull-up resistors on SCL and SDA. The accuracy for the $35-45^{\circ} \mathrm{C}$ interval is below $0.2{ }^{\circ} \mathrm{C}$ and the conversion time for 12 data bits is 220 ms typical.

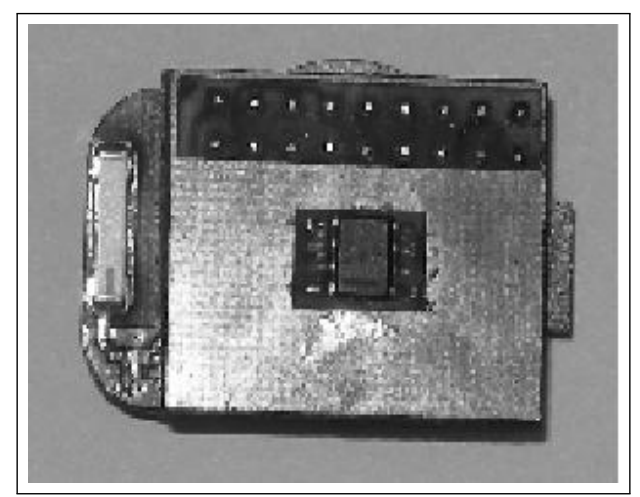

Figure 5: The thermometer module

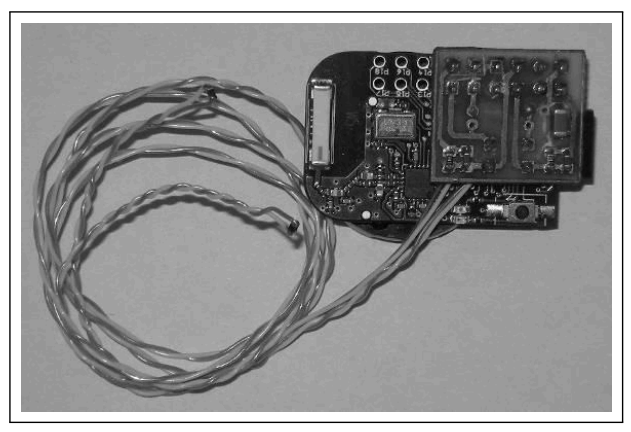

Figure 6: The respiration module

The Personal server samples the signal from the temperature sensor once per second and computes the status of the patient for the following temperature values: 
- Low temperature - when temperature falls below $35^{\circ} \mathrm{C}$;

- High temperature - when temperature rises above $38^{\circ} \mathrm{C}$;

- Normal temperature - between the above values.

The respiration module (Figure 6) uses one of the most usual methods to sense breathing to detect airflow using a nasal thermistor [9]. Although most applications require only breathing detection, some applications and diagnostic procedures require monitoring of the respiratory rhythm.

Our wireless respiration sensor uses a thermistor for long-time monitoring during the normal activity. The sensor is designed using MSP430F2274 microcontroller with an on-chip 10 bit A/D converter for data acquisition and CC2500 2.4GHz wireless transceiver. The thermistor detects changes of breath temperature between ambient temperature (inhalation) and lung temperature (exhalation). A thermistor placed in front of a nose detects breathing as a temperature change. The used thermistor is a 0603 SMD type and has the following characteristics: $R_{\text {nom }}=10 \mathrm{k} \Omega$ at $25^{\circ} \mathrm{C}, \mathrm{B}=3380,1 \%$ tolerance.

The respiration signals are recorded using the MSP430F2274 A/D converter with $10 \mathrm{~Hz}$ sampling frequency.

The Personal server on patient computes the following respiration parameters:

- Breathing amplitude - calculated for every breathing cycle as a difference between minimum (Inhalation) and maximum thermistor voltage (Exhalation);

- Breathing interval - measured between two minimums representing two inhalations;

- Breathing frequency calculated from the breathing interval as a number of breaths per minute. Normal breathing frequency is $12-20$ cycles/minute.

We consider two types of respiration:

- Normal respiration, when every breath lasts more than 0.5 seconds;

- Apnoea, when the breathing is missing for more than 10 seconds. Sleep apnoea can last more than 120 seconds.

The pulsoximeter sensor used is Micro Power Oximeter board from Smiths Medical [10] (Figure 7). The same sensor can be used for heart-rate detection and $\mathrm{SpO}_{2}$. The probe is placed on a peripheral point of the body such as a finger tip, ear lobe or the nose. The probe includes two light emitting diodes (LEDs), one in the visible red spectrum $(660 \mathrm{~nm})$ and the other in the infrared spectrum $(905 \mathrm{~nm})$. The percentage of oxygen in the body is computed by measuring the intensity from each frequency of light after it transmits through the body and then calculating the ratio between these two intensities.

The pulsoximeter communicates with the eZ430-RF2500 through asynchronous serial channel at CMOS low level voltages. Data provided includes $\% \mathrm{SpO}_{2}$, pulse rate, signal strength, plethysmogram and status bits and is sent to the eZ430-RF2500 at a baud rate of $4800 \mathrm{bps}, 8$ bits, one stop bit and no parity.

The Micro Power Oximeter has the following measurement specifications: range 0-99\% functional $\mathrm{SpO} 2$ (1\% increments), accuracy ą2 at 70-99\% $\mathrm{SpO}_{2}$ (less than $70 \%$ is undefined), pulse range 30-254 BPM (1 BPM increments), accuracy ą2 BPM or ą2\% (whichever is greater).

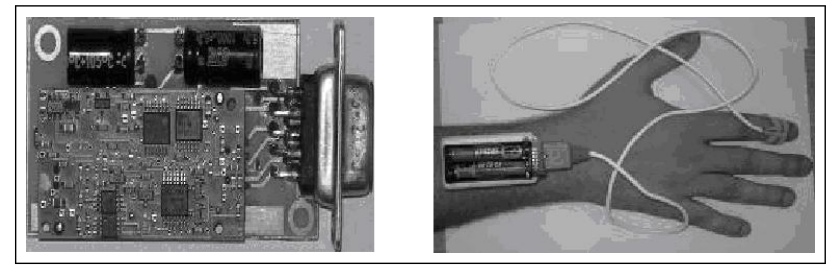

Figure 7: The pulsoximeter module 
For the blood pressure measurement, a commercially available A\&D UA-767PC BPM [11] was used. The blood pressure monitor (BPM) takes simultaneous blood pressure and pulse rate measurements. It includes a bi-directional serial port connection communication at $9600 \mathrm{kbps}$. An eZ430-RF2500 communicates with the BPM on this serial link to start the reading process and receives the patient's blood pressure and heart rate readings. Once the readings are received, the eZ430-RF2500 communicates with the network and transmits them to the Personal Server.

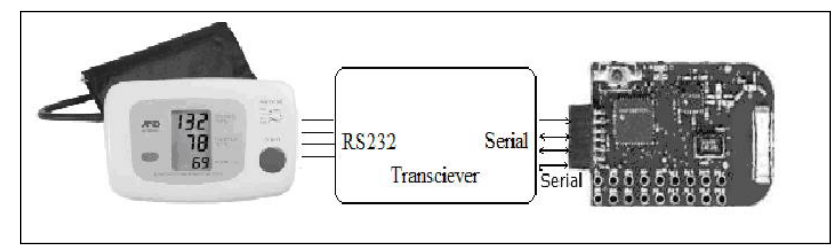

Figure 8: The blood pressure module (block diagram)

The Personal server computes blood pressure and defines the status of the patient by using the following blood pressure values:

- Hypotension: systolic $<90 \mathrm{mmHg}$ or diastolic $<60 \mathrm{mmHg}$;

- Normal: systolic 90-119mmHg and diastolic 60-79 $\mathrm{mmHg}$;

- Pre-hypertension: systolic 120-139 mmHg or 80-89 mmHg;

- Stage 1 Hypertension: systolic 140-159mmHg or diastolic 90 - $99 \mathrm{mmHg}$;

- Stage 2 Hypertension: systolic $\geqslant 160 \mathrm{mmHg}$ or diastolic $\geqslant 100 \mathrm{mmHg}$;

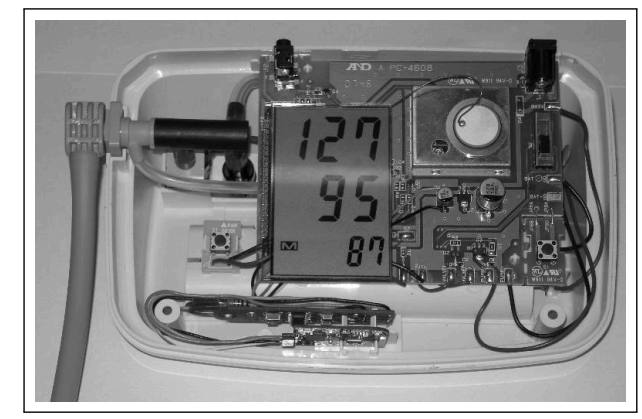

Figure 9: The blood pressure module

Our module for fall detection of humans is based on accelerometer technique. By using a tri-axial accelerometers our system can recognize patient movements. Linear acceleration are measured to determine whether motion transitions are intentional.

The algorithm for the human fall detection [3] uses the ADXL330 accelerometer and eZ430RF2500 Wireless Module. The ADXL330 is a small, thin, low power, complete three axial accelerometer with signal conditioned voltage outputs, all on a single monolithic IC. The product measures acceleration with a minimum full-scale range of ą3g. It can measure the static acceleration of gravity in tilt-sensing applications, as well as dynamic acceleration resulting from motion, shock, or vibration.

The microcontroler calculates the aA acceleration using the formula:

$$
a_{A}=\sqrt{a_{A_{x}}^{2}+a_{A_{y}}^{2}+a_{A_{z}}^{2}}
$$

We determine if the subject has fallen if the condition $a_{A}>0.4 \mathrm{~g}$ is valid. 


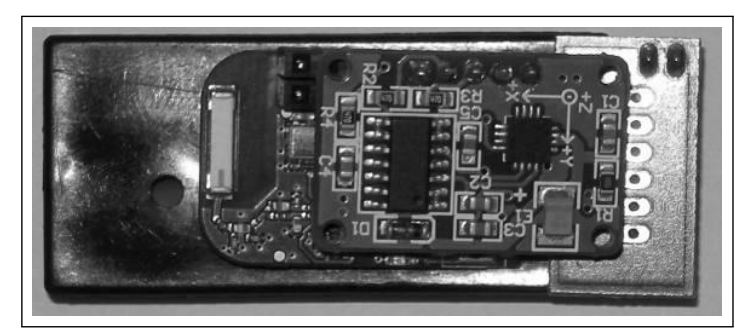

Figure 10: The fall detection module

\section{Results}

In the Figure 11 it is represented the personal server, that were implemented by means of a PDA (Fujitsu-Siemens Loox T830). This personal medical monitor is responsible for a number of tasks, providing a transparent interface to the wireless medical sensors, an interface to the patient, and an interface to the central server.

The USB interface (Figure 18) is realized by using a serial to USB transceiver (FT232BL) from FTDI [12] and enables eZ430-RF2500 to remotely send and receive data through USB connection using the MSP430 Application UART. All data bytes transmitted are handled by the FT232BL chip. It also contains a voltage regulator to provide $3.3 \mathrm{~V}$ to the eZ430-RF2500.

The software on the Personal Server [4], [5] receives real-time patient data from the sensors and processes them to detect anomalies.

The software working on the Personal Server (Figure 12) was written by using C\# from Visual Studio.NET, version 8. The software displays temporal waveforms, computes and displays the vital parameters and the status of each sensor (the battery voltage and distance from the Personal Server).

The distance is represented in percent of 100 computed based on RSSI (received signal strength indication measured on the power present in a received radio signal).

If the patient has a medical record that has been previously entered, information from the medical record (limits above the alarm become active) is used in the alert detection algorithm.

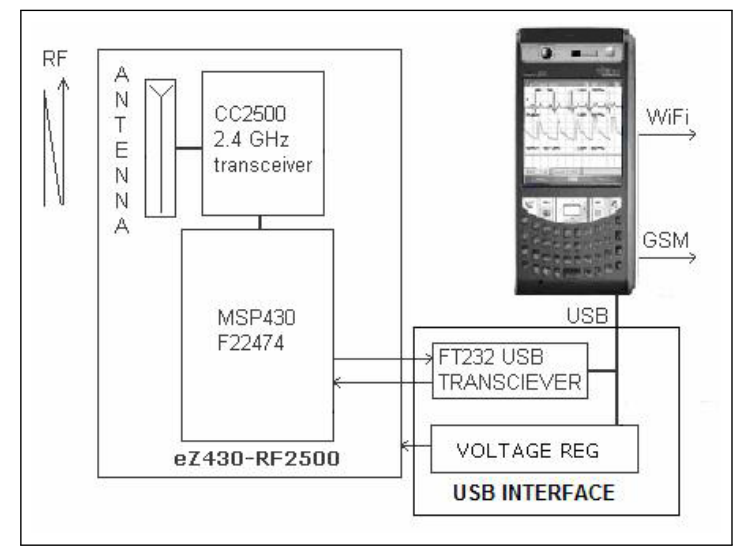

Figure 11: The Personal server (block diagram)

The following physiological conditions cause alerts:

- low $\mathrm{SpO} 2$, if $\mathrm{SpO} 2<90 \%$;

- bradycardia, if $\mathrm{HR}<40 \mathrm{bpm}$;

- tachycardia, if HR > 150bpm;

- HR change, if ?HR / 5 min $>20 \%$; 

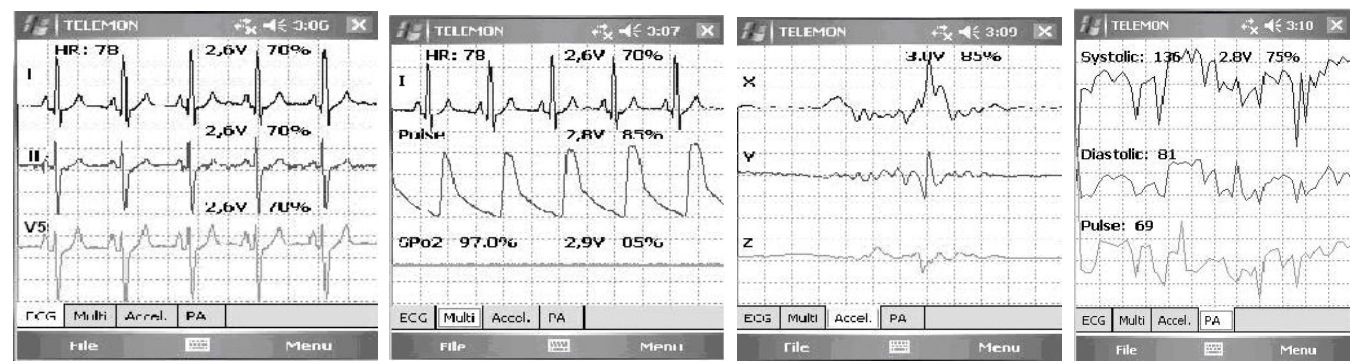

Figure 12: The Personal server interface: (a) 3 ECG traces, (b) one ECG trace, pulse waveform and $\mathrm{SpO} 2$, (c) 3 accelerometer traces, (d) systolic and diastolic pressure from BPM

- HR stability, if max HR variability from past 4 readings $>10 \%$;

- BP change if systolic or diastolic change is $>$ ą10\%.

When an anomaly is detected in the patient vital signs, the Personal server software application generates an alert in the user interface and transmits the information to the TELEMON Server.

\section{Summary and Conclusions}

In this paper it is presented a project that aims to develop a secure multimedia, scalable system, designed for medical consultation and telemonitoring services. The main goal is to build a complete pilot system that will connect several local telecenters into a regional telemedicine network. This network enables the implementation of teleconsultation, telemonitoring, homecare, urgency medicine, etc. for a broader range of patients and medical professionals, mainly for family doctors and those people living in rural or isolated regions.

The Regional Telecenter in Iasi, situated in the Faculty of Medical Bioengineering, will allow local connection of hospitals, diagnostic and treatment centers, as well as a local network of family doctors, patients, paramedics and even educational entities. As communications infrastructure, we aim to develop a combined fix-mobile-internet (broadband) links.

The proposed system will also be used as a warning tool for monitoring during normal activity or physical exercise.

Such a regional telecenter will be a support for the developing of a regional medical database, that should serve for a complex range of teleservices such as teleradiology, telepathology, teleconsulting, telediagnosis, and telemonitoring. It should also be a center for continuous training and e-learning tasks, both for medical personal and for patients.

\section{Acknowledgment}

This work is supported by a grant from the Romanian Ministry of Education and Research, within PN_II programme (www.cnmp.ro/Parteneriate), contract No. 11-067/2007.

\section{Bibliography}

[1] Costin H, Rotariu C. (2004) Processing and Analysis of Digital Images. Applications in Biomedical Imagistics, Tehnica-Info Publ. House, Kishinev, Rep. Moldova, 441 pp., ISBN 9975-63-196-7 
[2] European Commission, IST Directorate General (2006) Resource book of eHealth projects, Sixth Research and Development Framework Programme

[3] Giansanti D, et al, (2003) Is It Feasible to Reconstruct Body Segment 3-D Position and Orientation Using Accelerometric Data? IEEE Trans. On BME 50(4)

[4] Shorey R. (2006) Mobile, Wireless and Sensor Network: Technology, Applications and Future Directions, Ed. Wiley

[5] Wattenhofer R., (2005), Algorithms for ad hoc and sensor networks, Comp.Comm., 28, p.1498-1504

[6] Costin H, et al., (2006) A multimedia Telemonitoring Network for Healthcare. Enformatika, Transactions on Engineering, Computing and Technology, Vol. 17, Cairo, pp. 113-118

[7] Costin H, Morancea Octavia, et al. (2008) Integrated system for real time monitoring of patients and elderly people. Ukrainian Journal of Telemedicine and Medical Telematics, Vol. 6, No. 1 , pp. $71-75$

[8] Rotariu Cr., Costin H., Arotaritei D. and Constantinescu G. (2009) A Low Power Wireless Personal Area Network for Telemedicine, Proceedings of the 4th European Conference of the International Federation for Medical and Biological Engineering, Vol. 22, pp. 982-985

[9] Jovanov E., Raskovic D., Hormigo R., (2001) Thermistor-Based Breathing Sensor for Circadian Rhythm Evaluation, Biomedical Sciences Instrumentation, Vol. 37, pp. 493-497

[10] http://www.smiths-medical.com/Userfiles/oem/OEM.31392B1.pdf

[11] http://www.lifesourceonline.com/and_med.nsf/html/UA-767PC

[12] FT232 datasheet at http://www.ftdichip.com/FT232

[13] J. Pan and W.J. Tompkins, (1985), A real-time QRS detection algorithm, IEEE Trans. Biomed. Eng., vol. BME-32, pp. 230-236

[14] P.S. Hamilton and W.J. Tompkins, Quantitative investigation of QRS detection rules using the MIT/BIH arrhythmia database, IEEE Trans. Biomed Eng., vol. BME-33, pp. 1157-1165

[15] Tompkins WJ (ed.). (1993) Biomedical Digital Signal Processing: C-Language Examples and Laboratory Experiments for the IBM PC., Englewood Cliffs, NJ: PTR Prentice Hall

[16] P.S. Hamilton, (2002), Open Source ECG Analysis Software, E. P. Limited, Somerville, Mass, USA.

[17] MSP430 datasheet at http://www.ti.com/MSP430

[18] CC2500 datasheet at http://www.ti.com/CC2500 